\title{
An audit to assess knowledge about malnutrition among staff working in the UK SCI centres
}

\author{
S. S. Wong ${ }^{1,2}$, F. Derry ${ }^{1}$, G. Grimble ${ }^{2}$ and A. Forbes ${ }^{2}$ \\ ${ }^{1}$ National Spinal Injuries Centre, Stoke Mandeville Hospital, Aylesbury and ${ }^{2}$ Centre of Gastroenterology and Clinical \\ Nutrition, University College London Hospital \\ Correspondence: Samford Wong, email: Samford.Wong@buckshosp.nhs.uk
}

The importance of screening, identifying, treating and monitoring has been widely described in many international and national guidance. Recent UK-based multicentre study has identified that one in three patients with spinal cord injuries are at risk of malnutrition when admitted to the SCI centre ${ }^{(1)}$. Yet only $20 \%$ of these at-risk patients were referred for dietetic assessment and further intervention. Part of this is due to ignorance of the subject and its importance amongst, surgical trainees, for example ${ }^{(2)}$. We therefore decided to test the knowledge of malnutrition amongst doctors, nurses and dietitians in the SCI centre to see if it was a cause of the problem of underdiagnosis. The questionnaire aimed to test: (1) Awareness of nutrition screening tools and related care plans and; (2) Knowledge of doctors, nurses and dietitians working in the SCI centre.

One hundred and fifteen questionnaires were sent to 102 nurses, 17 doctors and 15 dietitians working in the UK SCI centres during January to March 2010. The questionnaires contain 14 multiple choice questions, with one in five possible answers considered correct, and there is an open question for ideas on improving nutrition screening in the SCI centre.

$62(46.3 \%)$ questionnaires were completed and returned for analysis. This includes 10 doctors, 39 nurses and 9 dietitians. The present audit demonstrated that awareness of the need for nutritional screening is good: $83 \%$ of staff reported they are aware there is a nutrition screening tool. This audit also demonstrated areas of poor knowledge such as calorie content of intravenous fluids, indicators of malnutrition and choice of nutritional support in malnourished patients. Although all doctors knew how to calculate BMI, only $38 \%$ of nurses know how to calculate BMI. Surprisingly, nearly half (49\%) of the participants thought that $20 \%$ weight loss or more was required to indicate malnutrition. This high perceived cut-off point, suggests that malnutrition is likely to continue to be undetected and unmanaged. The overall scores (median) showed clear differences in nutritional knowledge between groups (median; dietitians $92.8 \%$, doctors $53.5 \%$, nurses $35.7 \%, P<0.01$ or less. This suggests that dietitians have an important role in training health and social care professionals in nutrition.

This study highlights the need for further education in SCI medicine in order to improve the efficacy of feeding and nutrition therapy for SCI patients.

1. Wong SS, Derry F, Gandy J et al. (2010) Prevalence of malnutrition in patients admitted to UK Spinal Injury Centres - a multi centre study. Clin Nutr Suppl In press.

2. Awad S, Herrod PJ, Forbes W et al. (2010) Knowledge and attitudes of surgical trainees towards nutritional support: food for thought. Clin Nutr 29, 243-248. 